\title{
Low Grade Endometrial Stromal Sarcoma with Widespread Metastasis - A Rare Case Report
}

\author{
Neha Sethi*, C.L. Pande, Anjali Sharma and Kirti Pandia \\ Dept of Pathology Bhagwan Mahaveer Cancer Hospital and Research Centre, Jaipur, India
}

\section{ABSTRACT}

EST are rare tumors. According to WHO classification, they are divided into Endometrial stromal nodules (ESN), Low grade endometrial stromal sarcomas (LGESS) and Undifferentiated stromal sarcomas (USS) according to mitotic activity, vascular invasion and difference in prognosis. EST may be misdiagnosed as benign tumors especially in small biopsies. This may lead to increased risk of spread in patients at the time of final diagnosis. We report an interesting case of a premenopausal woman who presented with LGESS in a very widespread manner. In the present study, characteristic histopathology and IHC was really an adjunct to diagnose the case and a panel of antibodies put together like Caldesmon, SMA, PR, ER, Desmin and B-Catenin helped a lot to diagnose it.

\section{Keywords: Endometrial Stromal Tumors, CD 10, Metastasis}

\section{Introduction}

Endometrial stromal tumours (EST) account for $<10 \%$ of mesenchymal tumours of the uterus. They were first described by Norris and Taylor in 1966. ${ }^{[1]}$ According to WHO classification, they are divided into Endometrial stromal nodules (ESN), Low grade endometrial stromal sarcomas (LGESS) and Undifferentiated stromal sarcomas (USS) on the basis of mitotic activity, vascular invasion and difference in prognosis. These tumours typically consist of cells resembling endometrial stromal cells of the proliferative endometrium. LGESS account for approximately $0.2 \%$ of all malignant uterine tumours. ${ }^{[1]}$

EST occurs at 40 - 55 years of age group. They may present with abnormal uterine bleeding, pelvic pain and dysmenorrhoea or may be found incidentally. Distant metastasis occurs in about $15 \%$ of cases. ${ }^{[2]}$

Few of the cases show involvement of ovary at presentation. [3] So, while evaluating an ovarian tumour mimicking endometrial stromal tumour, uterine EST should be ruled out as they are more common.

\section{Case Report}

37 years female presented with lump abdomen along with pain since 15 days. The abdominal pain was associated with menstrual period and persisted for some days even after periods.

On examination, she had slightly raised serum CA 125 (261U/ml). On CECT abdomen, her right ovary was enlarged with presence of solid lesion measuring 46X42 $\mathrm{mm}$ along with presence of free fluid in Pouch of Douglas and multiple peritoneal deposits.
Fine needle aspiration cytology from right ovarian mass showed presence of malignant neoplasm. (FIGURE 1B)

Peritoneal nodule biopsy taken on diagnostic laproscopy, revealed presence of EST on microscopy. On immunohistochemistry, tumour cells were positive for CD 10 (Figure 1C), Progesterone receptor (PR) and Smooth muscle actin (SMA) while Desmin, S100 and CD 34 were negative ruling out the possibility of tumour of smooth muscle origin.

Patient was then given 3 cycles of chemotherapy and planned for Pan hysterectomy.

On gross examination, Cervix showed a grey firm area in the soft tissue adherent over its posterior aspect. A polypoidal projection was seen in fundus in uterus and rest of the endometrium was roughened.

Right ovary, peritoneal nodules, mesenteric nodules, appendix and omentum also showed grey firm areas measuring 0.5 to $0.8 \mathrm{~cm}$ in diameter.

Microscopically, polypoidal area at fundus showed presence of tumour. Rest of the endometrium showed necrotic changes and inflammation. Tumor was also seen infiltrating the soft tissue attached to the cervix (Figure 2B), peritoneal nodules, omentum, appendix (Figure 2D) and right ovary. Tumour showed densely cellular areas in which tumour cells are arranged in nodules, sheets and fascicles. The cells were uniform, spindle shaped with small round to oval nuclei and insconpicuous nucleoli and minimal atypia (Figure 1A). Cells were arranged radially around the small spiral arterioles. Mitosis was 
rare. Lymphovascular invasion was present in the form of intravascular worm like plugs in the myometrium (Figure 2A). Tumour was also seen infiltrating a subserosal fibroid (Figure 2C). No necrosis was identified associated with tumour. Final histological diagnosis was made as LGESS FIGO stage IIIB.
Patient condition was unremarkable post operatively. After 30 days of discharge, however patient was readmitted with complaints of pain abdomen. CT abdomen, was suggestive of ? post operative changes or ? Recurrence. However further confirmation was not done due to debilitated condition of patient.

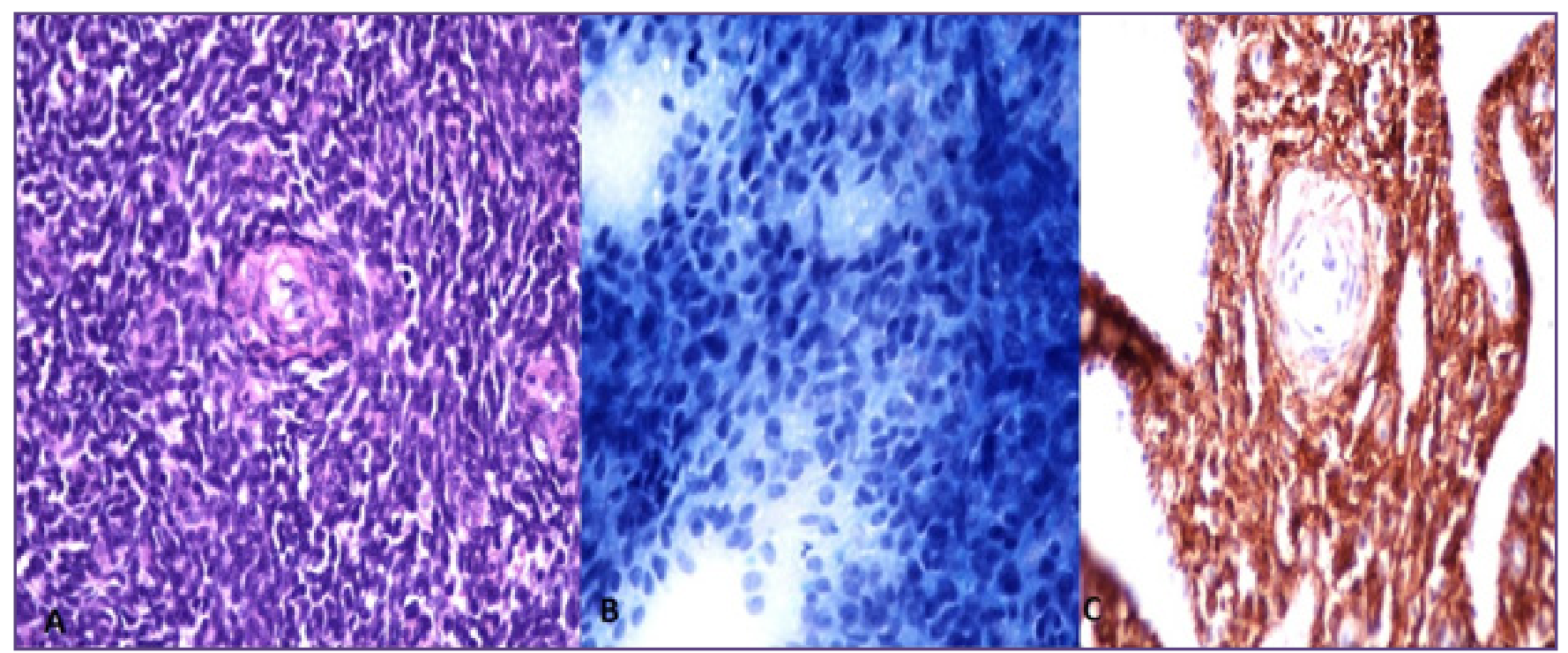

Fig. 1 A: H/E stained section of ESS (400X); B: FNAC from right ovarian mass (400X); C: CD 10 positive tumour cells (400X).

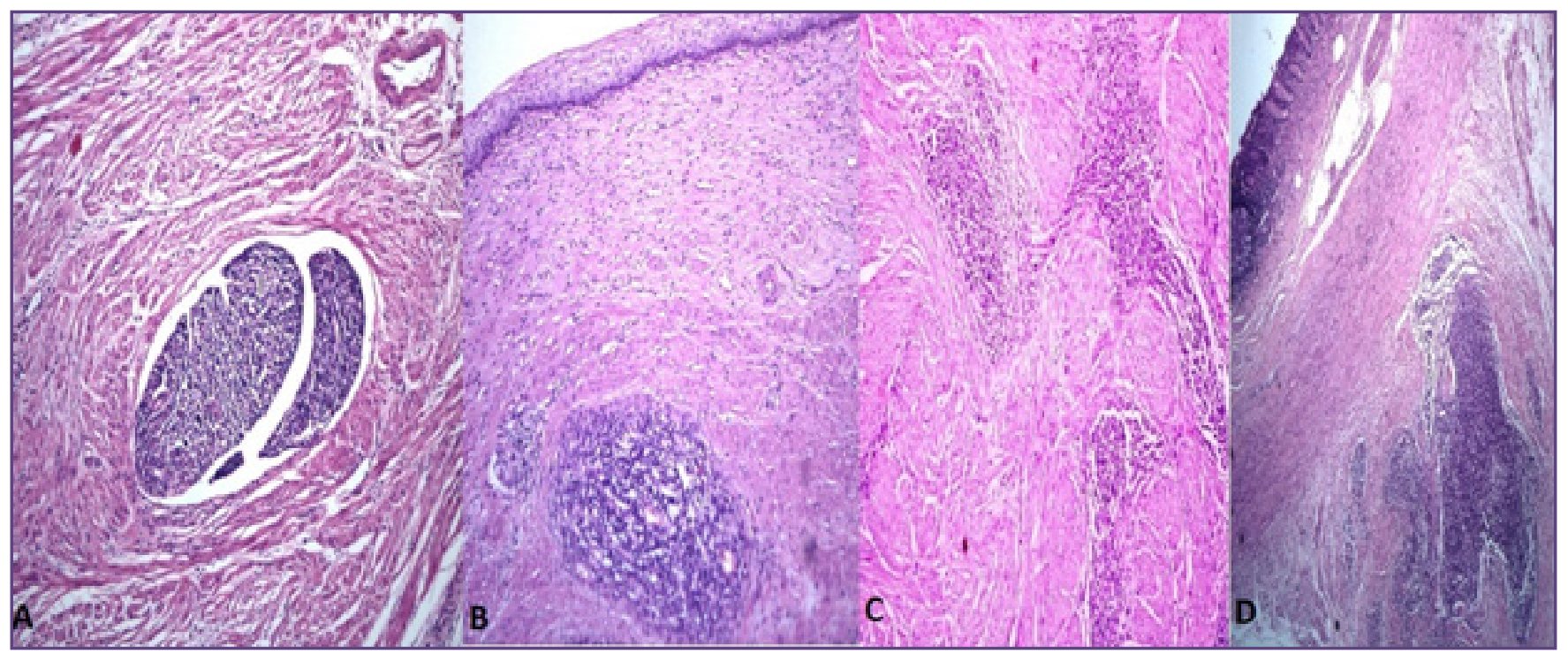

Fig. 2 A: H/E stained section of intravascular tumour emboli (100X); B: H/E stained section of tumour infiltrating cervix (100X); C: H/E stained section of tumour infiltrating subserosal fibroid (100X); D: H/E stained section of tumour infiltrating Appendix (40X).

\section{Discussion}

EST are rare tumours. EST may be misdiagnosed as benign tumours especially in small biopsies. This may lead to increased risk of spread in patients at the time of final diagnosis. Stemme et al in their study on 25 biopsy specimens have shown that most tumour fragments were $5 \mathrm{~mm}$ and larger and distinct from nearby fragments of benign stroma. ${ }^{[4]}$ Hence to identify stromal tumour, careful evaluation of endometrial stroma and background stroma should be made. 
Our patient was young, 37 years old with interesting clinical presentation of abdominal pain associated and persisting even after periods. Majority of cases present with abnormal uterine bleeding and abdominal pain or rarely as rapid enlargement of fibroid uterus. ${ }^{[5]}$ Most cases are perimenopausal with average age 45 years.

It's most common differential diagnosis is cellular Leiomyoma which show benign spindle shaped cells arranged in fascicles with whorling. They have large caliber vessels with thick muscular walls. The cells are desmin positive and CD 10 negative. ${ }^{[5]}$

It is also differentiated with intravenous leiomyomatosis however its morphological and immunohistochemical features are similar to conventional leiomyomas.

Most endometrial stromal tumours as well as normal endometrial stromal cells stain for CD10. But CD10 positivity is also seen in other tumour cells of smooth muscle origin, carcinosarcomas and rhabdomyosarcomas. Endometrial stromal tumours also show positivity for SMA and B-Catenin but are usually negative for Caldesmon and Desmin. Hence a panel of antibodies should be put together like Caldesmon, SMA, PR, ER, Desmin and B-Catenin to diagnose it. ${ }^{[6]}$ Also to exclude rare possibility of fibrous lesion CD 34 is used. In the present study, IHC was really an adjunct to diagnose the case.

At the molecular level genetic basis of endometrial stromal tumour is suggested as most cases show reciprocal chromosomal translocation $\mathrm{t}(7,17)(\mathrm{p} 15: \mathrm{q} 21)$ generating JAZF1-JJAZ1 fusion gene product detected by FISH and RT PCR. ${ }^{[7]}$

Other differential diagnosis includes low grade Mullerian Adenosarcomas and stromal adenomyosis. Low grade mullerian adenosarcomas have an admixture of epithelial and sromal elements. Epithelial component appears benign.

Another important differential include PECOMAs which may have a tongue-like infiltrative growth resembling the infiltrative pattern seen in low-grade ESS. However, tumour cells express human melanoma black 45, Melan-A or microphthalmia factor.

Surgery is the most effective treatment for ESS. As in our case, usually these tumours express ER and PR which makes them hormonally responsive. ${ }^{[8]}$ However various studies have shown that bilateral salpingo-oopherectomy do not affect overall survival in stage I ESS and in late stages hormone therapy may be done with or without tumour directed radiotherapy. ${ }^{[9]}$ In premenopausal young women, ovaries can be retained with stage I ESS.
Long term follow up is mandatory for the patients due to tendency for late recurrence. About $1 / 3^{\text {rd }}$ cases show recurrence within $10-15$ years. Metastases to the lungs and pelvic or peritoneal cavities are the most frequent. ${ }^{[2]}$ In another study, recurrence sites were found to be vagina, pelvis, and lung. However widespread metastasis including ovary, appendix, omentum and mesentry was noted in the present case.

At stage I, 5 years survival rate for ESS is $54 \%$ to $100 \%$ and stage II is $30 \%$. For stage III, IV it is only $11 \%$. Among its prognostic markers, tumour stage at diagnosis is most important. But tumour necrosis has some prognostic value according to Abeler et al. ${ }^{[10]}$ As seen in the present case, inspite of high degree of differentiation, minimal atypia and rare mitotic activity, disease was very widespread which showed their least importance as prognostic markers.

\section{Conclusion}

LGESS are very rare of all malignant uterine tumours. In cases of LGESS, such widespread and early metastasis is very rarely reported as was seen in the present case.

For improving survival of patient, proper evaluation of the case in terms of radiological and histopathological and immunohistological investigations should be done. It should be carefully diagnosed and differentiated from its benign differentials especially in small biopsies and young individuals for further management and follow up of cases.

\section{References}

1. Norris HJ, Taylor HB. Mesenchymal tumours of the uterus: I. A clinical and pathological study of 53 endometrial stromal tumours. Cancer. 1966; 19: 755-66.

2. Soliman, PT Lu, KH. "Neoplastic Diseases of the Uterus". In Lentz, GM; Lobo, RA; Gershenson, DM; Katz, VL. Comprehensive Gynecology (6th ed.). Mosby. ISBN 978-0-323-06986-1.

3. Zhu XQ, Shi YF, Cheng $X$, Zhao CL, Wu YZ. Immunohistochemical markers in differential diagnosis of endometrial stromal sarcoma and cellular leiomyoma. Gynaecol Oncol. 2004; 92:71-79.

4. Stemme S, Ghaderi M, Carlson JW. A clinicopathological study of 25 biopsy specimens with identification of problematic areas. Am J Clin Pathol. 2014; 141:133-9.

5. Fekete PS, Vellios F. The clinical and histologic spectrum of endometrial stromal neoplasms: a report of 41 cases. Int J Gynecol Pathol. 1984; 3: 198-212.

6. Angelo E D, Prat J. Uterine sarcomas: A review. Gynecologic Oncology. 2009, doi:10.1016/j.ygyno.2009.09.023

7. Micci F, Panagopoulos I, Bjerkehagen B, Heim S. Consistent rearrangement of chromosomal band $6 \mathrm{p} 21$ with generation of fusion genes JAZF1/PHF1 and EPC1/PHF1 in endometrial stromal sarcoma. Cancer Res. 2006; 66: 107-12. 
8. Landreat V, Paillocher N, Catala L, Foucher F, Descamps P, Leveque J. Low grade endometrial stromal sarcoma of the uterus; review of 10 cases. Anticancer research. 2008; 28: 2869-74.

9. Amant F, De Knijf A, Van CB, Leunen K, Neven P, Berteloot $\mathrm{P}$, et al. Clinical study investigating the role of lymphadenectomy, surgical castration and adjuvant hormonal treatment in endometrial stromal sarcoma. Br J Cancer 2007; 97: 1194-9.

10. Abeler VM, Royne O, Thorenson S, Danielsen HE, Nesland JM, Kristenson GB. Uterine sarcomas in Norway. A histopathological and prognostic survey of a total population from 1970 to 2000 including 419 patients. Histopathology. 2009;54:355-64

*Corresponding author:

Dr. Neha Sethi, Consultant, Pathology, Bhagwan Mahaveer Cancer Hospital and Research Centre, Jaipur, India

Phone: +91 9983947655

Email: nehasethi3@gmail.com

Financial or other Competing Interests: None. 